\title{
IDENTIFY CRITICAL DATA DURING PRODUCT CUSTOMISATION - A CASE STUDY OF ORTHOSES FABRICATION
}

\author{
X. Tan ${ }^{1, \otimes}$, W. Chen ${ }^{2}$, J. Cao ${ }^{2}$ and S. Ahmed-Kristensen ${ }^{3}$ \\ ${ }^{1}$ Imperial College London, United Kingdom, ${ }^{2}$ Xuzhou Central Hospital, China, ${ }^{3}$ Royal College of Art, United \\ Kingdom \\ $\triangle$ xinyang.tan14@imperial.ac.uk
}

\begin{abstract}
Big data provides high volume of data to inform product customisation. Understanding which data is relevant remains a challenge. A method is proposed to identify relevant data to inform datadriven customisation. A case study regarding customisation of orthoses was conducted. Verbal protocol analysis was employed to extract time spent on major fabrication phases. Data related to patients, therapists and fabrication time was analysed. Results showed that the number of stabilised joints, experience of therapists and whether the design is for in- or out-patient are key factors for customisation.
\end{abstract}

Keywords: human behaviour, protocol analysis, data-driven design, healthcare design, customisation

\section{Introduction}

Product customisation as a manufacture strategy fulfils specific customer requirements by transferring users' data into an established product platform with general production methods (Chen et al., 2005). Users' data can be gathered through variable methods, for instance surveys (Basoglu et al., 2009), onsite data collection (Ambrogio et al., 2005) and observation (Morrison et al., 2009), to extract individual customer requirements. This information can be employed in a general fabrication platform which also contains a lot of data from manufacturers or individual fabricators who perform the customisation, such as data regarding behaviours of fabricators (Barnes et al., 2006) and performance of a supply chain (Macchion et al., 2017). These data collected from both the end users and the manufacturers can be employed to optimise design and manufacture of customised products. Additionally, means of data collection have been increasingly adapted during product usage and fabrication. Sensors are embedded in products (Yang et al., 2007) and manufacture chains (Stock and Seliger, 2016), which enables a high volume of data acquisition for manufacturers. However, since information required for product development which defines parameters of products is usually shown as single values (dimensions, colours etc.), it is a challenge to narrow down and target the most valuable data (Klein et al., 2019). Thus, distinguishing, identifying and understanding crucial data with high influence on the customisation process from a large database is an essential for manufacturers, in order to customise their products efficiently.

Large quantity of data has been collected by researchers, to establish parameters for product development. User centred design (UCD) was performed in studies (Mata et al., 2017; Willard et al., 2018) by involving users into the design process and modifying design based on users' feedback 
regarding aesthetics and functions of products. Snowball sampling was applied in a UCD study (Dopp et al., 2019) that aims to enhance healthcare services in communities. The study covered nationally representative samples to achieve as much data as possible and a cluster analysis was conducted to categorise the data. In the study (Tao et al., 2018), a big amount of data collected from the entire manufacture chain and product lifecycle, which was processed and analysed to assist decision making and operation of the manufacture chain. Stavrokos et al. (2016) gathered ergonomic data from 200 head scans to inform the design of components for headsets in order to maximise comfort. They established an approach to cluster the data informing the number of variants needed. According to a big data framework regarding IoT home devices built by Jung et al. (2019), big data was analysed at the distribution, usage and repair stages of the product cycle to improve efficiency of each stage. From a research conducted by Voet et al. (2019), a method to capture valuable product usage data and applying machine leaning techniques on the data was demonstrated. Zheng et al. (2018) developed a data-driven approach to develop personalised smart, connected product with dense information flow in a cloud-based environment. A wearable mask was designed through co-development from both physical and cyber world. In the automotive industry, Tüchsen et al. (2018) applied a data-driven design approach (selection and generation mechanism) to deal with data of customers' requests and improved the efficiency of designing electric motors. Moreover, high volume of data was also obtained in studies (Ellena et al., 2018; Mourtzis et al., 2013), investigating mass customisation of products.

Although, large amount of data was collected in these studies, very few of them clearly demonstrated why the specific data was required and why the phase of product development or customisation was selected as the data source. Verification of the selected data and explanation of the ease of unessential data were not clearly explained, since a quantitative selection process was not yet sufficiently developed in literatures. In order to target the right place and time to collect less but only essential data, this study proposes a method (shown in Figure 1.) to identify critical data to be collected, specifically to inform customisation. The main principle of the method is to use Verbal Protocol Analysis (VPA) to extract quantitative data from the customisation process, so the correlation can be analysed between the customisation process and other input data from the two sources: end users and manufacturers. According to the correlation analysis result, unessential input data can be eliminated.

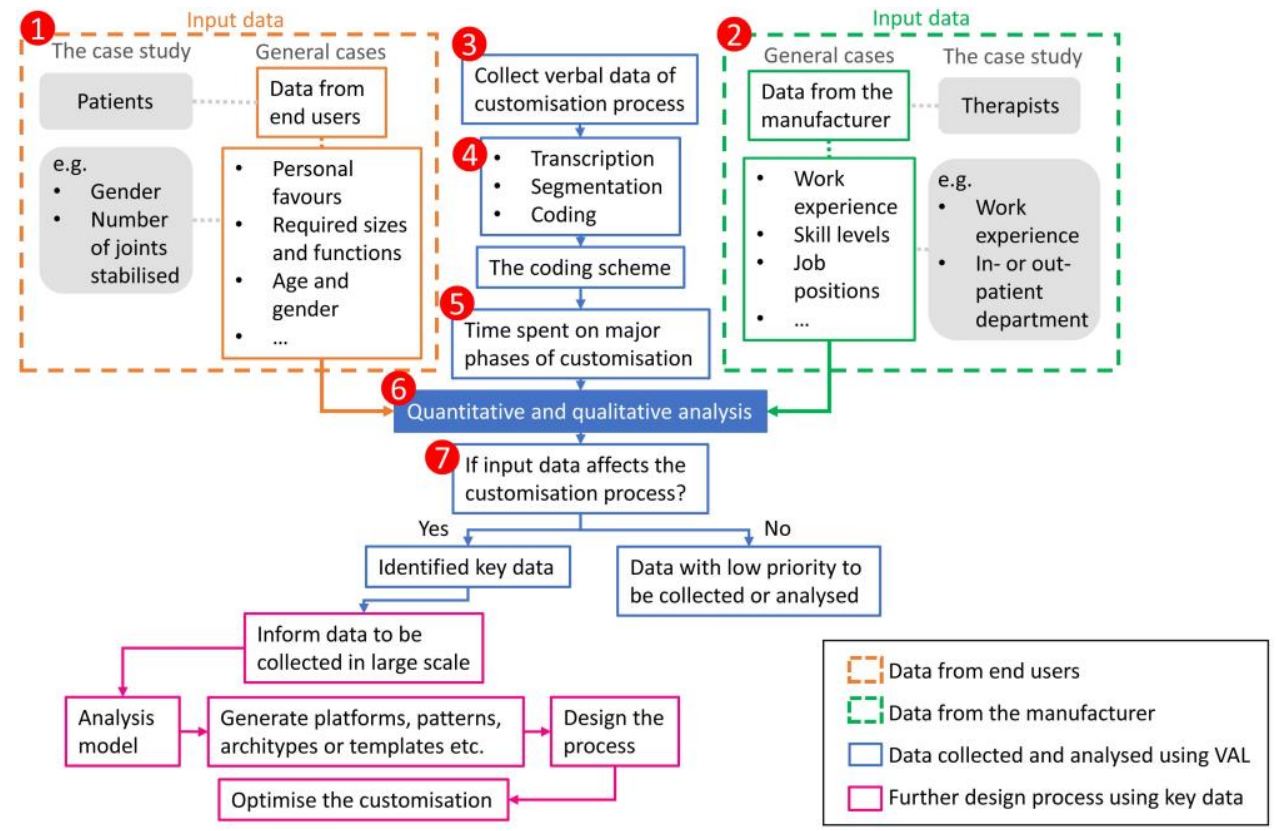

Figure 1. Method to identify key data

The data of end users can be their personal favours, age, gender, required sizes and functions etc. The data from the manufacturers can be fabricators' work experience, skill level, job positions etc. In the case study illustrated in this paper regarding the customisation of hand \& wrist (h\&w) orthosis, many data could be collected from patients (i.e. end users) and hand therapists (i.e. manufacturers). In order 
to indicate the method of identifying key data proposed in this paper, two types of data from patients and two types of data from hand therapists (referred to as therapists in this paper) were collected, which will be explained in the following sections.

The main purpose of applying VPA is to quantitatively disclose the implicit customisation process, including detailed actions and the decision-making process of fabricators. VPA has been commonly applied in studies to understand the decision-making process during performance of tasks using the Think-aloud technique (Ericsson and Simon, 1984; Charters, 2003). Regarding design and product development, the method can be conducted to extract user requirements, for instance to obtain deeper insights of user experience with wheelchairs from the literature (Wilkinson and De Angeli, 2014). Also, VPA has been applied to derive explicit design approaches from existing unclear design practices, such as designing conventional ceramics (Anwar et al., 2015), using traditional design tools (sketching and prototyping) (Bousseau et al., 2016), and understanding design expertise (Ahmed and Wallace, 2004). Thus, VPA was selected as the technique to reveal performances and intentions of fabricators. The customisation process can be quantified in terms of time consumption which assumes to be affected by critical input data.

In section 2, the procedure of conducting the Think-Aloud technique by therapists, the Protocol Analysis on verbal data and quantitative data analysis are demonstrated. The results of data analysis, qualitive explanations and limitations of the study are described in section 3 . Finally, section 4 provides concluding results and opportunities for future research.

\section{Methods}

A method with seven steps (see Figure. 1) is proposed here to identify crucial data that affects customisation: (1) Collecting data of end users (i.e. patients in the case study) which may be related to customisation, e.g. age, gender, personal favours, required sizes and functions etc.; (2) Collecting data from the manufacturer or the fabricators who conduct the customisation (i.e. therapists in the case study), e.g. work experience, skill levels, job positions, tools or techniques used for the job etc.; (3) Collecting verbal data of the fabricators during the customisation process using the Thinkaloud technique; (4) Processing the verbal data and generating the coding scheme; (5) Calculating time consumption of major activities reflected by the coding scheme; (6) Conducting statistical analysis with the data from end users, the data from the manufacturer and the data of time consumption; (7) according to results of the analysis, distinguishing which input data has more effective impact on the customisation process. By using the method, the correlation among individual requirements of customers, behaviours or decision-makings of fabricators and the customisation process can be quantitatively established, therefore, being able to compare the degree of dependency among seemingly related data.

The method was applied in a case study of observing the customisation process of h\&w orthoses for patients with hand injuries, including bone fractures, tendon injuries and mixed conditions. Low patient compliance of wearing orthoses has been reported due to side effects, including discomfort (Sandford et al., 2008) and pressure sores (Buurke et al., 1999), caused by poor customisations, and leading to more invasive interventions like surgeries (McKee and Rivard, 2004). The case study aims to identify relevant data for future research of improving the current orthoses customisation process. In this case, patients' gender was assumed to be one of impacts on customisation, due to (1) the difference of hand sizes between male and female may influence the customisation, and (2) gender was determined as a factor in some studies (Vig, 2012; Tardivel, 1998) in relation to compliance during rehabilitation treatments and patients' compliance may affect orthoses customisation. Also, to understand the complexity of the orthosis, the number of joints being stabilised by h\&w orthoses was considered as an approximate measure for complexity. The higher the number of injured segments of a hand, the more joints are required to be stabilised with more components of orthoses (Richard and Ward, 2005), which affects the customisation. Whether therapists were from in- or out-patient department and the number of years that these therapists have been working on fabricating h\&w orthoses were acquired as data from therapists. The verbal data was collected during the fabrication process of h\&w orthoses while a therapist was conducting the Think-aloud technique. The detailed study procedure is demonstrated in following sections. 


\subsection{Study setup}

The study was conducted in a clinical room of the Xuzhou Central Hospital. Data collection was conducted with four professional hand therapists (two from in-patient department and two from outpatient department) who have 11, 7, 16 and 8 years of working experience of customising orthoses respectively. They are referred as therapist 11 , therapist 7 , therapist 16 and therapist 8 in this paper according to their working experience. Consents of participation were received from the four therapists. Nine male and nine female hand-injured adult patients (eight out-patients and ten inpatients) who were prescribed a customised h\&w orthosis (made of low temperature thermoplastic (LTT)) were recruited. All patient participants were able to independently provide consents to join in the project. Patients with diabetes, hand paresthesia or loss of sensation on hands were excluded. Patients in whole day care and patients with mental illness or cognitive symptoms who could not independently provide consent to participate in the project were not recruited. The inclusion and exclusion criteria of patient recruitment were established collaboratively with therapists to avoid extreme cases which may be distinguished from general customisation and also to adhere to ethical processes. Patients joined the study voluntarily and they could withdraw at any time. For this study, all eligible participants consented to the study and completed the data collection process. The study has been approved by both Xuzhou Central Hospital Biomedical Research Ethics Committee and Imperial College Research Ethics Committee. All data was anonymously and confidentially stored, and care was taken to ensure that all participants, including therapists and patients, could not be identified.

\subsection{Data collection}

The full process of customising h\&w orthoses was filmed by a camera (Canon EOS 550D, Canon Inc.) which was set in a clinical room of the hospital. Only hands of therapists and patients were filmed in order to protect their identities. All therapists were asked to talk aloud their real-time thoughts during customising h\&w orthoses. Their voice and movements were recorded using the camera. Since the data collection was set in a public hospital with a real live task, rather than an artificial task in a lab setting, therapists could be interrupted by surrounding environment. Since all therapists are experienced and familiar with the task, interruptions were considered not affecting their performances. Interruptions were excluded from the verbal and observation data.

\subsection{Data processing}

The verbal protocol data was processed through four steps, including: 1) transcription, 2) segmentation, 3) coding, and 4) time calculation. Verbal data from videos was manually transcribed into written text. Interruptions during observations which were not relevant data to therapists' thoughts of performing the customisation task, for instance communicating with other hospital staff, patients and family members was not considered as eligible data and was removed during transcription. From the video recording, movements of therapists were captured, in some cases there was no accompanying verbal data obtained, as the experienced therapists were so familiar with their tasks that these movements could be completed automatically and unconsciously. It was observed, that when several movements were conducted intensively in a short time, it may be difficult for the therapists to express all their thoughts. In these cases, movements were transcribed as descriptive words and were later clarified and confirmed by therapists. Sometimes during observations, when new movements or tasks were observed while the therapist kept quiet, the researcher prompted and reminded the therapist to verbalise their actions, and if necessary, explain previous movements. These explanations were also transcribed.

The transcription was divided into segments based on two criteria: (1) If the verbal content was constantly related to a specific task or movement, consecutive sentences with same task content were categorised into the same segment, i.e. the intent was the same. (2) If the therapist described more than one tasks or movements in one speaking sentence, that sentence was separated into several parts corresponding to tasks. For example, the transcription 'I will stabilise the thumb metacarpophalangeal joint. Metacarpophalangeal joints of other four fingers should not be fixed. Not fixing them allows the four fingers to move when wearing the orthosis.' was separated into two parts. The first segment 'I will stabilise the thumb metacarpophalangeal joint' indicates the therapist's thoughts regarding 
defining the stabilised joint. But the second segment 'metacarpophalangeal joints of other four fingers should not be fixed. Not fixing them allows the four fingers to move when wearing the orthosis.' describes the therapist's decision of released joints. Thus, they were divided into two segments.

A coding scheme (Figure. 2) was generated with three levels of codes assigned to those segments. A bottom-up approach was used in the process as it helps constructing codes by deciphering segmented transcriptions (Narvaez, 1999) which was more suitable for this study. Feedback from the four therapists was sought to inform the coding scheme design. There are seven first level codes representing major phases and activities of the customisation process which were merged from nine second level and 38 third level codes. Third level codes demonstrating individual movement or tasks were generated by summarising each segment. Since a bottom-up approach was employed, as new third level code were revealed, these were added to the coding scheme and applied to review new transcriptions. Second level codes are middle level codes that bridges the first and third levels to help merging the third level codes. However, in some cases there were only two levels of codes. The code 'Explaining' is considered as an independent first level code as the hand therapist is communicating with a single intention of explaining to the researcher rather as part of their tasks.

\begin{tabular}{|c|c|c|}
\hline 1st level code & 2nd level code & 3rd level code \\
\hline \multicolumn{3}{|c|}{ Main Phases and Subordinate Activities of Fabrication } \\
\hline \multirow{11}{*}{ Preparation } & \multirow{2}{*}{ Condition evaluation } & Check diagnosis report \\
\hline & & Check X-ray image \\
\hline & \multirow{2}{*}{ Orthosis design } & Decide stabilised joints \\
\hline & & Decide released joints \\
\hline & \multirow{4}{*}{$\begin{array}{l}\text { Pre-fabrication } \\
\text { organisation }\end{array}$} & Organise gauze on the hand \\
\hline & & Organise tools for fabrication \\
\hline & & Position the hand \\
\hline & & Cover hand with cling film or fabric \\
\hline & \multirow{3}{*}{ LTT preparation } & Measure dimensions \\
\hline & & Cut LTT \\
\hline & & Soften LTT \\
\hline \multirow{8}{*}{ Shape forming } & \multirow{5}{*}{$\begin{array}{l}\text { Generate orthosis } \\
\text { form }\end{array}$} & Apply $L T T$ on the hand \\
\hline & & Cut $L T$ to fold the material \\
\hline & & Add enhancement structure \\
\hline & & Mould general shape \\
\hline & & Facilitate LTT hardening \\
\hline & \multirow{2}{*}{$\begin{array}{l}\begin{array}{l}\text { Cut off extra } \\
\text { material }\end{array} \\
\end{array}$} & Draw cutting references \\
\hline & & Cut off extra material \\
\hline & Re-softening & \\
\hline \multirow{7}{*}{ Adjustment } & \multirow{3}{*}{ Edge treatment } & Smoothen edges \\
\hline & & Bend or roll edges \\
\hline & & Cover edges with foam strips \\
\hline & \multirow{3}{*}{ Comfortable fitting } & Attach paddings on the hand \\
\hline & & Stick paddings on the orthosis \\
\hline & & Release pressure under the orthosis \\
\hline & \multicolumn{2}{|c|}{ Tries on patients during adjustment } \\
\hline \multirow{4}{*}{ Fastening } & \multirow{3}{*}{ Fasten Velcro } & Cut Velcro \\
\hline & & Attach Velcro \\
\hline & & Secure Velcro \\
\hline & \multicolumn{2}{|c|}{ Tries on patients during fastening } \\
\hline \multicolumn{3}{|c|}{ Interactions During Fabrication } \\
\hline \multirow{4}{*}{ Communicating } & \multicolumn{2}{|c|}{ Ask for patients' compliance } \\
\hline & \multicolumn{2}{|c|}{ Ask for patients' feedback } \\
\hline & \multicolumn{2}{|l|}{ Educate patients } \\
\hline & \multicolumn{2}{|c|}{ Answer patients' questions } \\
\hline \multirow{4}{*}{ Observing } & \multicolumn{2}{|c|}{ Check fitting and tightness } \\
\hline & \multicolumn{2}{|c|}{ Check ROM of released joints } \\
\hline & \multicolumn{2}{|l|}{ Check stabilised joints } \\
\hline & \multicolumn{2}{|l|}{ Check skin condition } \\
\hline Explaining & & \\
\hline
\end{tabular}

Figure 2. Coding scheme

The length of time spent for each activity described as the first level codes was calculated by summing the time spent on corresponding third level codes. The Figure. 3 shows the time spent on major phases and activities of one observation. The activity of 'Explaining' was scattered throughout the fabrication process while the therapist was explaining the reason for conducting a task to the researcher without actually performing movements in real time. Moreover, first level codes 'Observing' and 'Communicating' occurred at intervals during any of the fabrication phases and at the same time while other tasks were undertaken.

Differences of fabrication processes among therapists and between in and out-patient departments were quantitatively analysed. Qualitive evaluation has also been applied to explain the found correlation. 


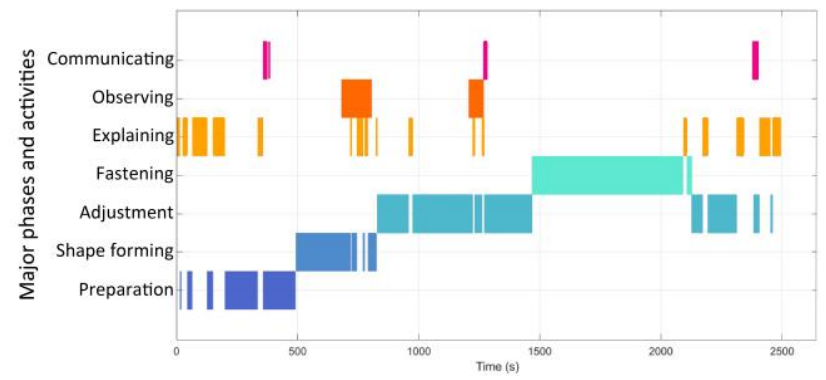

Figure 3. An example of time spent on major phases and activities of an observation

\section{Results and discussions}

\subsection{Number of stabilised joints}

As a $\mathrm{h} \& \mathrm{w}$ orthosis aims to stabilise affected joints and retain flexibility of unaffected joints, the number of joints that were stabilised by h\&w orthoses was assumed as the key data reflecting patients' requirements, thus would be needed for data driven customisation process for orthoses. For instance, three joints were stabilised of one observation with a single fracture at the 5th metacarpal bone. Whereas for another patient with multiple injured area including a finger amputation, bone fractures and tendon injuries, 13 joints were stabilised in various angles. Thus, this data is firmly related to patients' conditions. The mean number of stabilised joints of observations taken care by each therapist was shown in Figure. 4. It can be noted that, in-patients had more stabilised joints than out-patients, which indicated hypotheses that, (1) requirements for customisation between in- and out-patient are different, and (2) the number of joints to be stabilised by h\&w orthoses also influences the customisation. Therefore, these two types of data affect customisation and should be considered as priority for further research.

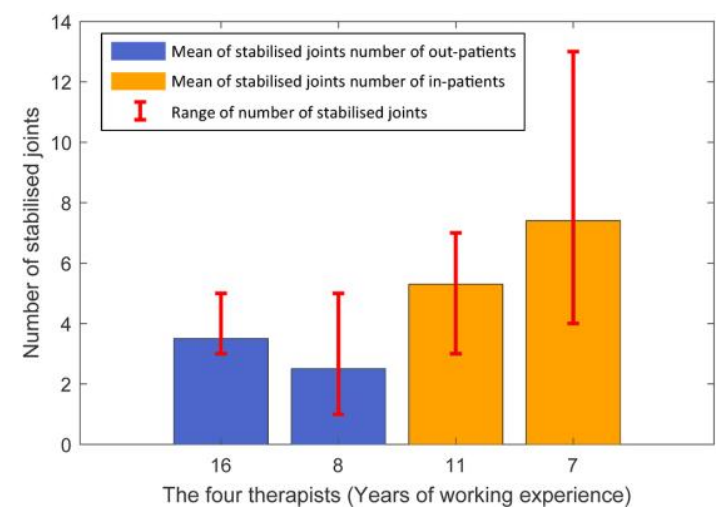

Figure 4. Number of joints stabilised by orthoses

\subsection{Total fabrication time and working experience of therapists}

In order to determine if the fabrication time was different among the four therapists in terms of their working experience, a one-way ANOVA test was conducted. The result showed that there was a significant difference on fabrication time consumed by therapists with different years of working experience $(\mathrm{p}=0.04)$. As shown in Figure 5, the greater the experience the therapist had, the more time was spent to customise a h\&w orthosis. Although this is somewhat surprising, and the number of therapists observed is too low to draw conclusion, the trend may be caused by two reasons: (1) more experienced therapists pay more attention to details, such as comfort and aesthetics, which take more time and may not be noticed by less experienced therapists; (2) therapists with more working experience may spend more time evaluating and refining completed work than less experienced therapists. This result indicated that some information was missed by less experienced therapists. In the further research of designing customised orthoses, this missing information will be clarified and highlighted for large scale data collection. 


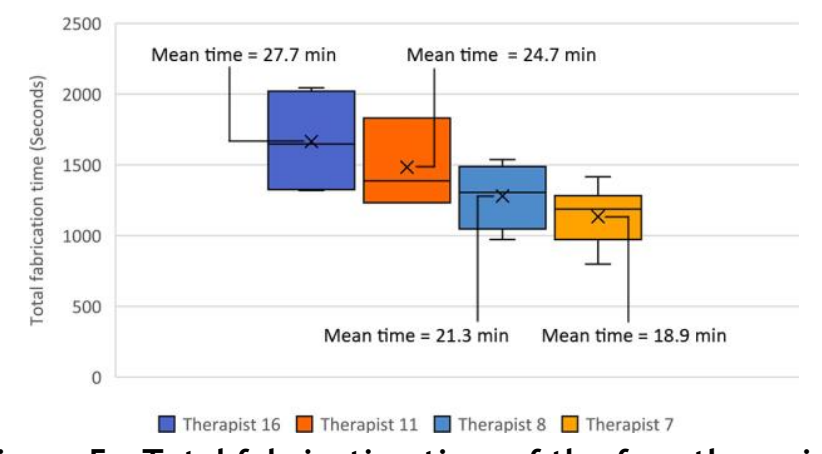

Figure 5. Total fabrication time of the four therapists

\subsection{Time spent on major phases by in- or out-patient therapists}

The time spent on the major phases of the customisation was quantitatively analysed and compared between therapists, to identify data of the therapist that influences the fabrication process. The four main phases were preparation, shape forming, adjustment and fastening as seen in Figure 2. The average percentage of time spent on the four major phases of fabrication conducted by each therapist was reported in Figure 6. The proportional arrangement of time was identical between therapist 16 and 8 (out-patient therapists), as well as between therapist 11 and 7 (in-patient therapists). Therapists 16 and 8 were found to spend the least proportional time on shape forming which are $15.45 \%$ and $13.55 \%$ respectively, followed by preparation and adjustment. They spent the highest proportion of fabrication time to fasten orthoses, $39.33 \%$ for therapist 16 and $39.86 \%$ for therapist 8 . For the in-patient therapists, shape forming occupied the largest percentage of their fabrication time, $36.60 \%$ for therapist 11 and $31.83 \%$ for therapist 7 . In contrast to the out-patient therapists, the in-patient therapists spent the least proportional time on fastening which were both $14.99 \%$.

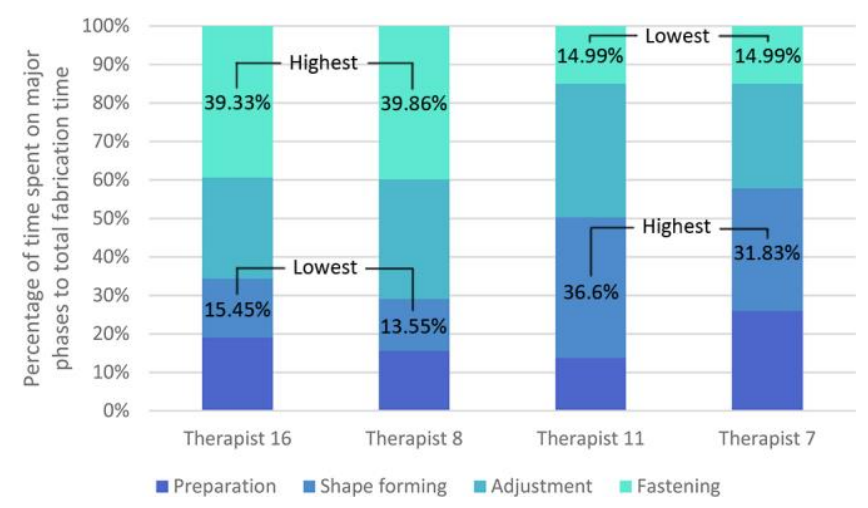

Figure 6. Proportional time spent on major phases of customisation

From these findings, we can see that the data and requirements between in- and out-patients differ impacting the fabrication process, thus this data is critical for any customisation of h\&w orthoses. For example, the therapists of the out-patient department paid more attention to fasten orthoses as the durability of orthoses is highly dependent on fasteners, and can be explained as out-patients will leave thus any further adjustments require new appointments. Thus, therapists need to ensure that fasteners have been secured successfully without fear of losing tightness once patients have left hospital. Shape forming took more time for in-patients, this maybe due to two reasons: (1) In-patients who recently completed their surgeries require more care for stabilisation and fit as open wounds and implanted nails for bone fixation are taken into consideration during the shape forming phase. (2) As more joints were required to be stabilised for in-patients than out-patients which was demonstrated in section 3.1, the shape of orthoses formed by in-patient therapists are more complex to involve more joints with various angles. The result confirms that the number of joints to be stabilised by $h \& w$ orthoses was a key data affecting the customisation and distinguishes fabrication process between in- and out-patient therapists. Moreover, since patients living in the hospital have easy access to repair or reinstall by inpatient therapists. Fastening, in this case, is not the priority during orthoses fabrication. 
These results will inform data collection for future research related to design or optimisation of orthosis customisation. For instance, in- and out-patient customisation should be considered separately, though they apply similar customisation processes with same techniques and materials. Specifically, data collection for in-patient customisation should focus on methods that improves the efficiency of shaping complex forms of orthoses, whereas, data related to solutions of ensuring durability should be highlighted in the case of out-patient.

\subsection{Gender of patients}

The total fabrication time was compared between male and female patients using t-test which revealed no significant difference between male and female subjects $(\mathrm{p}>0.1)$. The mean of percentage of total fabrication time spent on major phases of customisations for male and female patients are reported in Figure 7. It can be seen from the figure that no significant difference of time spent on major phases can be identified. Time was mostly spent on the phase adjustment and the least time was arranged for preparation with observations of both genders. Therefore, in this case, gender of patients should not be involved as crucial data as it did not affect the performance of therapists regarding time consumption.

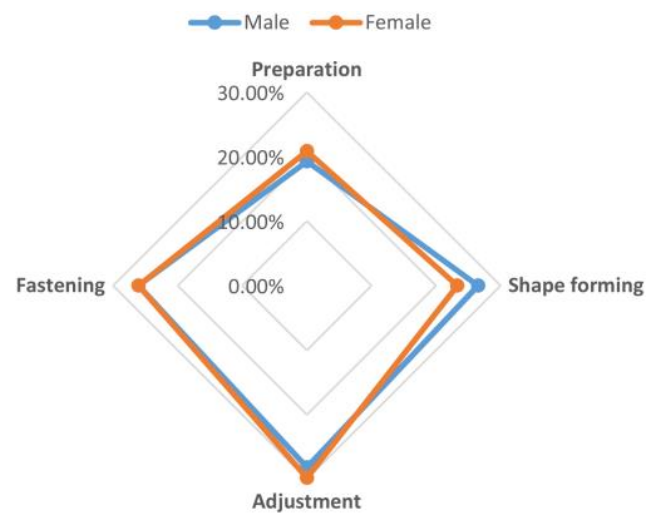

Figure 7. Compare proportional time spent on major phases between male and female patients

\subsection{Limitations}

The case study recruited 18 patients and 4 professional therapists in order to assess which data informs the customisation process of h\&w orthoses. Although, data regarding number of stabilised joints of patients and whether patients were in- or out-patient were identified as effective factors with influences on orthoses fabrications, due to the intensity of the verbal protocol analysis method, data was collected from a relatively small sample size which cannot represent all fabrications of h\&w orthoses. To understand this better, collaboration with multiple hospitals with a large number of observations would enhance the validity of the collected data.

A qualitive evaluation was conducted by the researcher collaboratively with therapists to select the mostly related data from all possible data that could be acquired from patients and therapists. Feedback from professionals were essential, which reduced the efficiency of the method and iterations of analysis occurred before crucial data could be identified. However, once a type of data can be defined as a key factor influencing the customisation process, it will facilitate data acquisition for future studies. As part of further research of this case study, more types of data from patients and therapists will be applied in the method to reveal and verify key factors, and findings inform the type of data that is needed for this.

\section{Conclusion}

A method was proposed in this paper to identify critical data influencing the process of product customisation. Statistical analysis was employed on data from end users and fabricators, revealing their correlation with time consumption of major phases and activities during the product development which was explicated using verbal protocol analysis. This method can be used to better understand the type of data that is relevant to customise products. A case study related to the customisation of hand and wrist 
orthoses for patients with hand injuries was conducted. The method was used to evaluate if (1) the number of joints to be stabilised, (2) patients' genders, (3) working experience of therapists and (4) in- or out-patients that therapists work with, impact the customisation in terms of time consumption of major phases and activities during the process. The results of the qualitive and quantitative analysis showed that the number of joints to be stabilised by orthoses, the number of years of therapists fabricating orthoses and whether patients were in- or out-patients were identified as key factors influencing the customisation process, whereas the gender of the patient was not relevant. As part of further study, more input data will be analysed through the method to reveal more critical factors.

Contribution of the method is the identification of critical data, for researchers and manufacturers alike, who are developing data-driven approaches to inform customisation which involves manual fabrication, for instance, manufacturers of customised clothes, bespoke wearable devices and customdesigned machines etc. The input data (data inside the orange and green squares in Figure 1) may be different from case to case. Compared with existing methodologies, e.g. using cluster analysis to reduce data quantity collected (Dopp et al., 2019; Stavrokos et al., 2016), this method filters out unessential data prior to investing in the collection of big data. Also, it can quantitatively interpret the implicit process of high degree customisation to facilitate further design and optimisation.

\section{Acknowledgement}

The study was conducted in the Royal College of Art, the Imperial College London and the Xuzhou Central Hospital. The project was partially supported by the China Scholarship Council, the Imperial College London and the Xuzhou Central Hospital. The procedure of the study has been approved by the Xuzhou Central Hospital Biomedical Research Ethics Committee (XZXY-LJ-20190222-003) and the Imperial College Research Ethics Committee (19IC5128). The authors would like to thank all participants who have contributed to this research.

\section{References}

Ahmed, S. and Wallace, K.M. (2004), "Understanding the knowledge needs of novice designers in the aerospace industry”, Design studies, Vol. 25 No. 2, pp. 155-173. https://doi.org/10.1016/j.destud.2003.10.006

Ambrogio, G. et al. (2005), "Application of Incremental Forming process for high customised medical product manufacturing", Journal of Materials Processing Technology, Vol. 162, pp. 156-162. https://doi.org/ 10.1016/j.jmatprotec.2005.02.148

Anwar, R., Abidin, S.Z. and Hassan, O.H. (2015), "A framework of empirical study through design practice for industrial ceramic sanitary ware design”, In International Colloquium of Art and Design Education Research (i-CADER 2014), Springer, Singapore, pp. 683-694. https://doi.org/10.1007/978-981-287-332-3_70

Barnes, L. et al. (2006), "A mass customised supply chain for the fashion system at the design-production interface", Journal of Fashion Marketing and Management: An International Journal. https://doi.org/ $10.1108 / 13612020610679312$

Basoglu, N., Daim, T.U. and Sofuoglu, E. (2009), "A decision methodology for customising software products", International Journal of Industrial and Systems Engineering, Vol. 4 No. 5, pp. 554-576. https://doi.org/ 10.1504/IJISE.2009.024158

Bousseau, A. et al. (2016), "How novices sketch and prototype hand-fabricated objects", In Proceedings of the 2016 CHI Conference on Human Factors in Computing Systems, ACM, pp. 397-408. https://doi.org/ $10.1145 / 2858036.2858159$

Buurke, J.H. et al. (1999), "Usability of thenar eminence orthoses: report of a comparative study", Clinical rehabilitation, Vol. 13 No. 4, pp. 288-294. https://doi.org/10.1191/026921599671603077

Charters, E. (2003), "The use of think-aloud methods in qualitative research an introduction to think-aloud methods", Brock Education: A Journal of Educational Research and Practice, Vol. 12 No. 2. https://doi. org/10.26522/brocked.v12i2.38

Chen, C.H., Khoo, L.P. and Yan, W. (2005). "PDCS - a product definition and customisation system for product concept development", Expert Systems with Applications, Vol. 28 No. 3, pp. 591-602. https://doi.org/ 10.1016/j.eswa.2004.12.040

Dopp, A.R. et al. (2019), "Integrating implementation and user-centred design strategies to enhance the impact of health services: protocol from a concept mapping study", Health research policy and systems, Vol. 17 No. 1, p.1. https://doi.org/10.1186/s12961-018-0403-0

Ellena, T. et al. (2018), "A design framework for the mass customisation of custom-fit bicycle helmet models", International Journal of Industrial Ergonomics, Vol. 64, pp. 122-133. https://doi.org/10.1016/j.ergon.2018.01.005

Ericsson, K.A. and Simon, H.A. (1984), Protocol analysis: Verbal reports as data. the MIT Press. 
Jung, J.J., Kim, K. and Park, J. (2019), "Framework of Big data Analysis about IoT-Home-device for supporting a decision making an effective strategy about new product design", In 2019 International Conference on Artificial Intelligence in Information and Communication (ICAIIC), IEEE, pp. 582-584. https://doi.org/ 10.1109/icaiic.2019.8669086

Klein, P. et al. (2019), "Towards an approach integrating various levels of data analytics to exploit product-usage information in product development", In Proceedings of the Design Society: International Conference on Engineering Design, Cambridge University Press, pp. 2627-2636. https://doi.org/10.1017/dsi.2019.269

Macchion, L., Fornasiero, R. and Vinelli, A. (2017), "Supply chain configurations: a model to evaluate performance in customised productions", International Journal of Production Research, Vol. 55 No. 5, pp. 1386-1399. https://doi.org/10.1080/00207543.2016.1221161

Mata, M.P. et al. (2017), "Investigating the influence of product perception and geometric features", Research in Engineering Design, Vol. 28 No. 3, pp. 357-379. https://doi.org/10.1007/s00163-016-0244-1

McKee, P. and Rivard, A. (2004), "Orthoses as enablers of occupation: client-centred splinting for better outcomes", Canadian Journal of Occupational Therapy, Vol. 71 No. 5, pp. 306-314. https://doi.org/ 10.1177/000841740407100510

Morrison, C. and Blackwell, A.F. (2009), "Observing end-user customisation of electronic patient records", In International Symposium on End User Development, Springer, Berlin, Heidelberg, pp. 275-284. https://doi.org/10.1007/978-3-642-00427-8_16

Mourtzis, D., Doukas, M. and Psarommatis, F. (2013), "Design and operation of manufacturing networks for mass customisation”, CIRP Annals, Vol. 62 No. 1, pp. 467-470. https://doi.org/10.1016/j.cirp.2013.03.126

Narvaez, D. (1999), "Using discourse processing methods to study moral thinking", Educational Psychology Review, Vol. 11 No. 4, pp. 377-393. https://doi.org/10.1023/a:1022061417089

Richard, R. and Ward, R.S. (2005), "Splinting strategies and controversies", The Journal of burn care \& rehabilitation, Vol. 26 No. 5, pp. 392-396. https://doi.org/10.1097/01.bcr.0000176886.63559.8b

Sandford, F., Barlow, N. and Lewis, J. (2008), "A study to examine patient adherence to wearing 24-hour forearm thermoplastic splints after tendon repairs", Journal of Hand Therapy, Vol. 21 No. 1, pp. 44-53. https://doi.org/10.1197/j.jht.2007.07.004

Stavrakos, S.K., Ahmed-Kristensen, S. and Goldman, T. (2016), "Using archetypes to create user panels for usability studies: Streamlining focus groups and user studies”, Applied ergonomics, Vol. 56, pp. 108-116. https://doi.org/10.1016/j.apergo.2016.02.016

Stock, T. and Seliger, G. (2016), "Opportunities of sustainable manufacturing in industry 4.0", Procedia Cirp, Vol. 40, pp. 536-541. https://doi.org/10.1016/j.procir.2016.01.129

Tao, F. et al. (2018), "Data-driven smart manufacturing”, Journal of Manufacturing Systems, Vol. 48, pp. 157169. https://doi.org/10.1016/j.jmsy.2018.01.006

Tardivel, J. (1998), "Gender differences in relation to motivation and compliance in cardiac rehabilitation", Nursing in critical care, Vol. 3 No. 5, pp. 214-219.

Tüchsen, J. et al. (2018), "Data driven design selection and generation-an industrial case study on electric motors”, In DS 92: Proceedings of the DESIGN 2018 15th International Design Conference, pp. 1709-1720. https://doi.org/10.21278/idc.2018.0223

Vig, K.W. (2012), "Patient compliance to wear orthodontic retainers during postretention may vary by age, gender, and time since braces were removed", Journal of Evidence Based Dental Practice, Vol. 12 No. 1, pp. 35-36. https://doi.org/10.1016/s1532-3382(12)70037-4

Voet, H. et al. (2019), “A Framework for the Capture and Analysis of Product Usage Data for Continuous Product Improvement”, Journal of Manufacturing Science and Engineering, Vol. 141 No. 2, p. 021010. https://doi.org/10.1115/1.4041948

Wilkinson, C.R. and De Angeli, A. (2014), “Applying user centred and participatory design approaches to commercial product development", Design Studies, Vol. 35 No. 6, pp. 614-631. https://doi.org/10.1016/ j.destud.2014.06.001

Willard, S. et al. (2018), "Development and testing of an online community care platform for frail older adults in the Netherlands: a user-centred design", BMC geriatrics, Vol. 18 No. 1, p. 87. https://doi.org/10.1186/ s12877-018-0774-7

Yang, X. et al. (2007), “A component-based software framework for product lifecycle information management for consumer products", IEEE Transactions on Consumer Electronics, Vol. 53 No. 3, pp. 1195-1203. https://doi.org/10.1109/tce.2007.4341604

Zheng, P., Xu, X. and Chen, C.H. (2018), “A data-driven cyber-physical approach for personalised smart, connected product co-development in a cloud-based environment", Journal of Intelligent Manufacturing, pp. 1-16. https://doi.org/10.1007/s10845-018-1430-y 